\title{
Sustained co-delivery of ibuprofen and basic fibroblast growth factor by thermosensitive nanoparticle hydrogel as early local treatment of peri-implantitis
}

This article was published in the following Dove Medical Press journal:

International Journal of Nanomedicine

\author{
Wenlei Chen' \\ Min Zhi' \\ Zujian Feng ${ }^{2}$ \\ Pengfei Gao' \\ Yuan Yuan' \\ Congcong Zhang' \\ Yonglan Wang' \\ Anjie Dong² \\ 'Department of Periodontology, \\ School and Hospital of Stomatology, \\ Tianjin Medical University, Tianjin, \\ People's Republic of China; \\ ${ }^{2}$ Department of Polymer Science \\ and Technology and Key Laboratory \\ of Systems Bioengineering of the \\ Ministry of Education, School \\ of Chemical Engineering and \\ Technology, Tianjin University, Tianjin, \\ People's Republic of China
}

Correspondence: Yonglan Wang Department of Periodontology, School and Hospital of Stomatology, Tianjin Medical University, Qixiangtai Road No 12, Tianjin 300070,

People's Republic of China

Tel +86222333209l

Email 732794I72@qq.com

Anjie Dong

Department of Polymer Science and Technology and Key Laboratory of Systems Bioengineering of the Ministry of Education, School of Chemical Engineering and Technology, Tianjin University, Weijin Road No 92, Tianjin, 300072, People's Republic of China

Tel +862227403536

Email ajdong@tju.edu.cn
Objective: The aims of this study were to 1) encapsulate ibuprofen (IBU) and basic fibroblast growth factor (bFGF) in a thermosensitive micellar hydrogel, 2) test the biological properties of this in situ drug delivery system, and 3) study the effect of hydrogel in promoting soft tissue healing after implant surgery and its anti-inflammatory function as an early local treatment of peri-implantitis.

Materials and methods: A thermosensitive micellar hydrogel was prepared from amphiphilic copolymer poly( $\varepsilon$-caprolactone-co-1,4,8-trioxa [4.6]spiro-9-undecanone)-poly(ethylene glycol)poly( $\varepsilon$-caprolactone-co-1,4,8-trioxa [4.6]spiro-9-undecanone) (PECT) nanoparticles and tested in vitro using a scanning electron microscope, rheometer, UV spectrophotometer, HPLC, and transmission electron microscope.

Results: The bFGF + IBU/PECT hydrogel formed a stable, water-dispersible nanoparticle core shell that was injectable at room temperature, hydrogel in situ at body temperature, and provided sustained release of both hydrophilic and hydrophobic drugs. The hydrogel promoted the proliferation and adhesion of human gingival fibroblasts, upregulated the expression of adhesion factors such as vinculin proteins, and showed anti-inflammatory properties.

Conclusion: In situ preparation of IBU- and bFGF-loaded PECT hydrogel represents a promising drug delivery system with the potential to provide early local treatment for peri-implantitis Keywords: thermosensitive hydrogel, nanoparticles, dual drugs, peri-implantitis

\section{Introduction}

Titanium dental implants are widely used clinically to rehabilitate patients affected by edentulism $;^{1}$ however, peri-implantitis significantly influences the success of implant restoration. ${ }^{2}$ Current methods for preventing and treating peri-implantitis include 1) the use of a modified titanium surface such as anatase, ${ }^{3}$ 2) recommending a 6-month follow-up with complementary sessions provided by a professional oral hygienist, 3) use of photodynamic therapy, ${ }^{4}$ and 4) bone regeneration. A previous study reported that epithelial cell proliferation is weaker around the implant than adjacent to the natural tooth. ${ }^{5}$ Moreover, tissue regeneration is difficult to establish once periimplantitis sets in. Although surgery can effectively remove the lesions and direct tissue reconstruction around the implants, the treatment can be traumatic and economically demanding. Therefore, it is important to prevent inflammation around implants and/or treat the inflammation early if it does occur during restoration. 
Recent evidence suggests that the long-term survival of osseointegrated implants depends on implant collar healing and stability. Coronal soft tissue attachment to the implant forms a protective closure, preventing bacterial invasion and inflammation. ${ }^{6,7}$ Therefore, soft tissue closure is essential for stable osseointegration and long-term implant survival..$^{8-10}$ Clinicians and patients must focus on the maintenance and recovery of soft tissue around the implant. Basic fibroblast growth factor (bFGF) is a potent pro-angiogenic factor with various biological effects that may be important in periodontal regeneration. bFGF induces angiogenesis, cell proliferation and differentiation, increases extracellular matrix production, and creates favorable conditions for periodontal soft tissue regeneration. ${ }^{11}$ However, it is readily hydrolyzed enzymatically. Its half-life in the human body is only 3-10 minutes, and its utilization rate is very low. Therefore, it is necessary to find a suitable protective carrier material for bFGF.

Pharmaceutical carrier materials consist mainly of natural (chitosan, liposome, collagen, etc) and synthetic (polylactic acid, polyethylene glycol [PEG], poly[lactic acid-glycolic acid] complex, etc) high-molecular weight polymers. Natural carrier materials are highly biocompatible, but they are costly, vary widely among batches, and are easily cross-contaminated. In contrast, the production of synthetic materials is controllable, inexpensive, and can be performed on a large-scale. Moreover, they induce no antigen reactions. However, as organic solvents are often used in the production of synthetic materials and to embed drugs, polypeptide or protein drug embedding is either ineffective or the drugs lose their activity during fabrication.

Sustained-release gel is easy to use and can be administered by ordinary syringes and needles. The solid polymer formed after the injection easily takes on the irregular shape of the periodontal pocket, and the patient adjusts well to it. Currently, it has become the main research direction for sustained-release preparations for periodontal disease. The novel thermosensitive hydrogel carrier amphiphilic copolymer poly( $\varepsilon$-caprolactone-co-1,4,8-trioxa [4.6]spiro9-undecanone)-poly(ethylene glycol)-poly( $\varepsilon$-caprolactoneco-1,4,8-trioxa [4.6] spiro-9-undecanone) (PECT) possesses many unique characteristics. PECT hydrogel requires neither high-temperature treatment nor low-temperature quenching, which are necessary to prepare PEG/polycaprolactone hydrogel. Moreover, PECT can load various bioactive compounds such as hydrophilic and hydrophobic drugs, proteins, peptides, fluorescent molecules, and others. The drug release rate can be appropriately controlled by altering the components of the hydrogel. Because it undergoes a thermosensitive sol-to-hydrogel phase transition, PECT can be stored at room temperature, requires no pretreatment, and forms stable hydrogels in vivo.

We predict that PECT hydrogel loaded with bFGF will provide sustained drug release and prolong bFGF half-life. We also expect that PECT will protect bFGF activity so that the drug can fully exert its biological effects. We intend to use this sustained drug release system to promote the proliferation and adhesion of human gingival fibroblasts (HGFs) while inhibiting inflammation. Microorganisms accumulated in the plaque around implants or at the edge of the mucosa cause local inflammation, and the inflammatory cells release a large number of cytokines causing tissue damage, which in turn results in degradation of connective tissue and subsequent bone destruction. Therefore, we introduced ibuprofen (IBU) into this system to reduce local prostaglandin, leukotriene, and IL levels, mitigate gingival inflammation, and inhibit alveolar bone resorption. We performed a series of experiments to detect drug release and hydrogelation behavior in vitro and to evaluate the effects of the hydrogel in promoting the proliferation and adhesion of HGFs and in inhibiting inflammation. These properties should help reduce the risk for peri-implant mucositis and treat it at the early stages.

\section{Materials and methods Materials}

bFGF was obtained from PeproTech (Rocky Hill, NJ, USA). IBU was purchased from Hunan Pharmaceutical Excipient Engineering Technology Research Center Co. Ltd. (Hunan, People's Republic of China). Porphyromonas gingivalis lipopolysaccharide (Pg-LPS) was purchased from InvivoGen (San Diego, CA, USA). PEG (Mn =1,500), $\varepsilon$-caprolactone (CL), and stannous octoate were obtained from Shanghai Aladdin Bio-Chem Technology Co. Ltd. (Shanghai, People's Republic of China). 1,4,8-trioxa [4.6] spiro-9-undecanone (TOSUO) was prepared in our laboratory according to a published procedure. ${ }^{12}$ Tetrahydrofuran (THF) and dimethyl sulfoxide were purchased from Concord Co. Ltd. (Tianjin, People's Republic of China). PECT was prepared from PEG, CL, and TOSUO using ring-opening copolymerization method as reported previously. ${ }^{13}$

\section{Preparation and characterization of PECT hydrogel loaded with bFGF and IBU}

PECT hydrogel was loaded with IBU and bFGF in two stages. First, freeze-dried PECT-IBU powder was fabricated by nanoprecipitation. Then, IBU and PECT were dissolved in THF, slowly mixed, and then gradually added dropwise to double distilled water. The mixture was magnetically stirred 
at room temperature for 12 hours to remove THF. Next, IBU-loaded nanoparticle dispersion was freeze-dried into a powder and dissolved in PBS while bFGF was added. The mixture was magnetically stirred for $4-6$ hours. The final product was PECT hydrogel aqueous dispersion loaded with both IBU and bFGF (bFGF + IBU/PECT).

The sol-hydrogel-sol transition was determined by the test tube inverting method. Vials (capacity $2.0 \mathrm{~mL}$ and diameter $1.1 \mathrm{~cm}$ ) containing $1.0 \mathrm{~mL}$ bFGF + IBU/PECT nanoparticle aqueous dispersion was immersed in a water bath at $5^{\circ} \mathrm{C}$ for 20 minutes. Changes were observed and noted based on flow (sol)-no flow (hydrogel) standards.

The PECT and bFGF + IBU/PECT hydrogels were flashfrozen in liquid nitrogen and lyophilized for 72 hours. The freeze-dried hydrogels were fractured, and their interior morphologies were observed by scanning electron microscopy ([SEM], S-4800; Hitachi Ltd., Tokyo, Japan).

Rheological measurements of aqueous PECT and bFGF + IBU/PECT nanoparticle dispersions were performed under oscillatory and steady shear conditions with a fluid rheometer (MCR 302; Anton Paar, Graz, Austria) set to an automatic gap. PECT and bFGF + IBU/PECT nanoparticle aqueous dispersions $(25 \% \mathrm{w} / \mathrm{v})$ were placed between parallel plates (diameter $=25 \mathrm{~mm}$ ) separated by $1.0 \mathrm{~mm}$. The storage modulus $\left(\mathrm{G}^{\prime}\right)$ and loss modulus $\left(\mathrm{G}^{\prime \prime}\right)$ were determined at $0.01 \mathrm{~Pa}$ and $1.0 \mathrm{~Hz}$, respectively. The heating rate was $1^{\circ} \mathrm{C} \mathrm{min}$.

\section{In vitro drug release}

The release of bFGF was simulated with BSA-fluorescein isothiocyanate conjugate (FITC). bFGF + IBU/PECT and BSA-FITC + IBU/PECT aqueous dispersions were placed in $1.0 \mathrm{~cm}$ (inner diameter) tubes and incubated at $37^{\circ} \mathrm{C}$ for 1 hour to form stable hydrogels. These hydrogels were then added to $4 \mathrm{~mL}$ PBS ( $\mathrm{pH} \mathrm{7.4)} \mathrm{at} 37^{\circ} \mathrm{C}$ in a constant temperature oscillator $(100 \mathrm{rpm})$. At predetermined times, $3 \mathrm{~mL}$ supernatant aliquots were removed and replaced with equal volumes of fresh medium. Three parallel samples were taken from each group. IBU in the supernatant was evaluated by HPLC (Lab Alliance Model 201; Thermo Fisher Scientific, Waltham, MA, USA) fitted with a Hypersil ${ }^{\mathrm{TM}}$ ODS-2 $(250 \times 4.6 \mathrm{~mm} \times 5 \mu \mathrm{m}) \mathrm{C} 18$ column (Thermo Fisher Scientific) at $230 \mathrm{~nm}$. BSA-FITC was measured by UV spectrophotometry (Beckman Coulter, Brea, CA, USA). The concentrations of IBU and BSA-FITC were calculated using a standard curve. The accumulated drug release was calculated according to the following formula:

$$
E=\left(\frac{V_{\mathrm{E}} \sum_{1}^{n-1} C i+V_{0} C n}{m_{0}}\right) \times 100 \%
$$

$\mathrm{E}$ is the cumulative release; $V_{\mathrm{E}}$ is the sampling volume; $V_{0}$ is the initial release volume; $C i$ and $C n$ is the drug concentration; $\mathrm{i}$ and $\mathrm{n}$ are the number of samples; $\mathrm{m}_{0}$ is initial mass of drug in the gel.

During the in vitro drug release experiment, the nanoparticle solution released from the hydrogel was collected at days 6,12 , and 18 . The size and distribution of the nanoparticles were characterized by dynamic light scattering (DLS). The morphology of the nanoparticles was measured by dropping them onto copper grids (400 mesh), dried at room temperature, and examined under transmission electron microscopy ([TEM], JEM-2100F; JEOL, Tokyo, Japan).

\section{HGF culture}

Primary HGFs were cultured until passages 4-6, at which time they were used in subsequent in vitro cell experiments. The study was approved by the Institutional Ethics Committee of the Tianjin Medical University and conformed to the regulations of the Declaration of Helsinki. All participants provided written informed consent. The details are described in the Supplementary materials.

\section{Biocompatibility of PECT}

PECT cytotoxicity tests were conducted in vitro on HGFs by using cell counting kit-8 (CCK-8) (Dojindo Laboratories, Kumamoto, Japan) reagent. Cells were seeded in 96-well plates at an initial density of $1 \times 10^{4}$ per well. After 24 hours, various PECT concentrations were added. There were five replicates per group. The well contents were replaced with serum-free DMEM $(100 \mu \mathrm{L})$ and CCK-8 solution $(10 \mu \mathrm{L})$ after 48 hours and incubated at $37^{\circ} \mathrm{C}$ for 4 hours. The absorbance of all samples was measured with a microplate reader (Varioskan Flash; Thermo Fisher Scientific) at $450 \mathrm{~nm}$.

\section{Cell proliferation assay of bFGF}

To optimize the bFGF concentration promoting HGF growth, a CCK-8 assay was performed with HGFs seeded in various bFGF concentrations for 1, 3, 5, and 7 days. The details are described in the Supplementary materials.

\section{HGF proliferation assay}

Titanium disks were placed in a 48 -well culture plate, and HGFs were seeded onto their surfaces at $1 \times 10^{3}$ per well. After 24 hours, the cells were randomly divided into the following groups: 1) growth medium (GM) (DMEM with

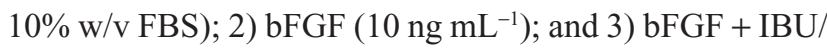
PECT hydrogel. Three replicate wells were used per group. After 48 hours, cells attached to the disks were washed with PBS, fixed in $4 \%$ formaldehyde, and dehydrated with an 
ethanol concentration series $(30 \%, 50 \%, 70 \%, 80 \%, 90 \%$, $95 \%$, and $100 \% \mathrm{v} / \mathrm{v}$ ) for 10 minutes per concentration. The cells were critical point dried and deposited on 10-nm thick gold-palladium for SEM observation.

The bFGF + IBU/PECT hydrogel proliferation was evaluated by CCK-8. Three experimental groups were used as described above. Cells were seeded in 96-well plates at an initial density of $1 \times 10^{3}$ per well. There were three replicates per group. Medium was replaced after 24 hours and again every 3 days. On days $1,3,5$, and 7 , cell samples were gently washed with PBS; the medium in each well was replaced with serum-free DMEM $(100 \mu \mathrm{L})$ and CCK-8 solution $(10 \mu \mathrm{L})$, and incubated at $37^{\circ} \mathrm{C}$ for 4 hours. Absorbances were measured with a microplate reader at $450 \mathrm{~nm}$.

The long-term effects of hydrogel on HGF growth were verified using Transwell chambers. The bFGF + IBU/PECT hydrogel was placed in a Transwell chamber, which was then set on the edge of a 24 -well plate and incubated at $37^{\circ} \mathrm{C}$ for 12 hours to form a stable hydrogel. HGFs were seeded in a 24-well plate at a density of $1 \times 10^{4}$ per well and incubated in $1 \mathrm{~mL}$ medium for 24 hours. There were three groups each with three replicates: group A, blank (control) GM; group B, $10 \mathrm{ng} \mathrm{mL} \mathrm{mL}^{-1} \mathrm{bFGF}$; and group C, bFGF + IBU/PECT hydrogel placed in the 24-well Transwell (polyethylene terephthalate; pore size $8.0 \mu \mathrm{m}$; EMD Millipore, Billerica, MA, USA) set over the cell culture plates. The culture medium was changed every 3 days, but the hydrogel of the Transwell chamber was not. A 24-well plate containing CCK-8 was sampled on days $1,3,5$, and 7 to measure cell viability by using a microplate reader.

\section{HGF adhesion assay}

Three types of smooth titanium disks (1 mm thick; 10, 14.75, and $33.9 \mathrm{~mm}$ diameter; TA1; Baoti, Shaanxi, People's Republic of China) were polished with silicon carbide sandpaper (No 1500 grit series). All samples were ultrasonically washed with acetone, absolute ethanol, and double distilled water for 30 minutes per treatment. The specimens were dried at room temperature for 1 hour, and then autoclaved at $120^{\circ} \mathrm{C}$ for 20 minutes before use.

HGFs were seeded onto titanium disks placed in 24-well plates and cultured in DMEM containing 10\% w/v FBS. Medium was replaced after 24 hours. The experimental groups were the same as those used in SEM analysis of cell proliferation experiment. After 48 hours, the samples were fixed in $4 \% \mathrm{w} / \mathrm{v}$ paraformaldehyde, permeabilized with $0.05 \%$ w/v Triton X-100 (Sigma-Aldrich Co., St Louis, MO, USA), and blocked in BSA solution. The primary antibody was rabbit anti-vinculin (Abcam, Cambridge, UK) and the secondary antibody was FITC-conjugated rabbit anti-IgG (Abcam). Cells were incubated with anti-vinculin antibody (1:100 dilution) overnight at $4{ }^{\circ} \mathrm{C}$. The cells were then rinsed with PBS and exposed to secondary antibody (1:200 dilution) for 1 hour. The nuclei were stained with DAPI (Sigma-Aldrich Co.). Images were captured with an inverted fluorescence microscope (Olympus Corporation, Tokyo, Japan).

Quantitative real-time (qRT)-PCR was performed to investigate the effects of bFGF + IBU/PECT hydrogel on vinculin gene expression. HGFs were inoculated on 33.8-mm titanium disks for 24 hours. All media were replaced after 24 hours. The experimental groups were the same as those described above. After 48 hours, total RNA was isolated with RNAiso Plus (TaKaRa Bio Inc., Shiga Prefecture, Japan). RNA concentration and purity were determined with Nanodrop ND 1000 Spectrophotometer (Thermo Fisher Scientific). The RNA was reverse transcribed into cDNA with a reverse transcription reagent kit (TaKaRa Bio Inc.) according to the manufacturer's instructions. qRT-PCR was performed with Maxima SYBR Green/ROX qPCR (Thermo Fisher Scientific) and QuantStudio 6 Flex (Thermo Fisher Scientific). Gene expression was calculated by the $2^{-\Delta \Delta \mathrm{Ct}}$ method. The sequences and genes used in this study are listed in Table 1. GAPDH was used as a reference gene standard.

\section{Inflammatory cytokine expression}

HGFs were seeded in 6-well plates at a density of $5 \times 10^{5}$ per well. All media were replaced when the cells reached $80 \%$ confluence. The cultures were randomly assigned to one of the four groups: 1) GM (DMEM containing 10\% FBS); 2) $1 \mu \mathrm{g} \mathrm{mL} \mathrm{m}^{-1}$ Pg-LPS; 3) $1 \mu \mathrm{g} \mathrm{mL} \mathrm{mL}^{-1} \mathrm{Pg}-\mathrm{LPS}+60 \mu \mathrm{g} \mathrm{mL}{ }^{-1} \mathrm{IBU}$; and 4) $1 \mu \mathrm{g} \mathrm{mL} \mathrm{m}^{-1} \mathrm{Pg}-\mathrm{LPS}+\mathrm{bFGF}+\mathrm{IBU} / \mathrm{PECT}$ hydrogel. After $10,24,48$, and 72 hours, the media was removed and centrifuged at 3,000 rpm for 20 minutes. Supernatants were extracted for ELISA prostaglandin E2 (PGE2) detection with a human ELISA kit (slmH, Shanghai, People's Republic of China) following the manufacturer's protocol.

\section{Statistical analysis}

All quantitative data were expressed as mean \pm SD. Statistical analysis of the results was performed using one-way

Table I Primer sequences for RT-PCR

\begin{tabular}{|l|l|}
\hline Gene & Primer sequence $\mathbf{( 5}^{\prime} \mathbf{-} \mathbf{3}^{\prime} \mathbf{)}$ \\
\hline \multirow{3}{*}{ Vinculin } & F:GATGCTGGTGAACTCAATGA \\
\cline { 2 - 2 } & R:CGAATGATCTCGTTAATCTC \\
\hline \multirow{2}{*}{ GAPDH } & F:TTCATTGTGGGAGCAGAC \\
\cline { 2 - 2 } & R:CAGCAGTTTCTCCAGAGC \\
\hline
\end{tabular}

Abbreviation: RT, real-time. 
ANOVA and the least significant difference test. $P<0.05$ was considered significant and $P<0.01$ was considered highly significant.

\section{Results}

\section{Characterization of thermosensitive behaviors of bFGF- and IBU-loaded PECT hydrogel}

Test tube inversion demonstrated that the bFGF + IBU/ PECT dispersion hydrogel at $25^{\circ} \mathrm{C}$ and $37^{\circ} \mathrm{C}$, as shown in Figure 1A. Therefore, bFGF and IBU encapsulation had no significant effect on the thermosensitive hydrogels.

Figure 1B shows SEM images of hydrogel morphology. Both PECT and bFGF + IBU/PECT hydrogels had a uniform, continuous porous structure. The pores in the hydrogel structure enabled media to flow through and transport drugs.

To elucidate the phase transitions in bFGF + IBU/PECT hydrogel, we measured changes in storage modulus $\left(\mathrm{G}^{\prime}\right)$ and loss modulus $\left(\mathrm{G}^{\prime \prime}\right)$ in the PECT and bFGF + IBU/PECT aqueous nanoparticle solutions as functions of temperature from $20^{\circ} \mathrm{C}$ to $55^{\circ} \mathrm{C}$ (Figure 2). As shown in Figure 2, $\mathrm{G}^{\prime \prime}$ sharply increased with temperature. At that point, the hydrogel was formed. The dual drug-loaded bFGF + IBU/PECT hydrogel had a larger modulus than the blank PECT hydrogel. IBU loading may have increased the hydrophobicity of the PECT nanoparticle and hydrogel network.

\section{In vitro IBU and BSA-FITC release profiles}

Figure 3 shows the time profile of IBU and BSA-FITC release from the hydrogel. The drug-loading efficiencies were 1 and $2 \mathrm{mg} \mathrm{mL}^{-1}$, respectively. The drug release curve showed that sustained IBU release lasted for 4 weeks and the cumulative release was $\leq 85 \%$. The drug release was slow, the trend was stable, and there was no obvious release burst. The BSAFITC release rate was higher than that of IBU. It proceeded gradually in a nearly linear manner until day 10 .

To reveal the in vitro drug release and degradation of $\mathrm{bFGF}+\mathrm{IBU} / \mathrm{PECT}$ hydrogel, the released medium was monitored by DLS and TEM. Figure 4 shows that the bFGF + IBU/PECT hydrogel underwent surface erosion as it degraded. TEM confirmed that the nanoparticles released from the hydrogel were spherical, $110 \mathrm{~nm}$ in diameter, and had a size distribution of 0.189 in aqueous solution.

\section{In vitro PECT biocompatibility}

Biocompatibility is essential in biomaterial applications. After HGFs were co-cultured in various PECT concentrations
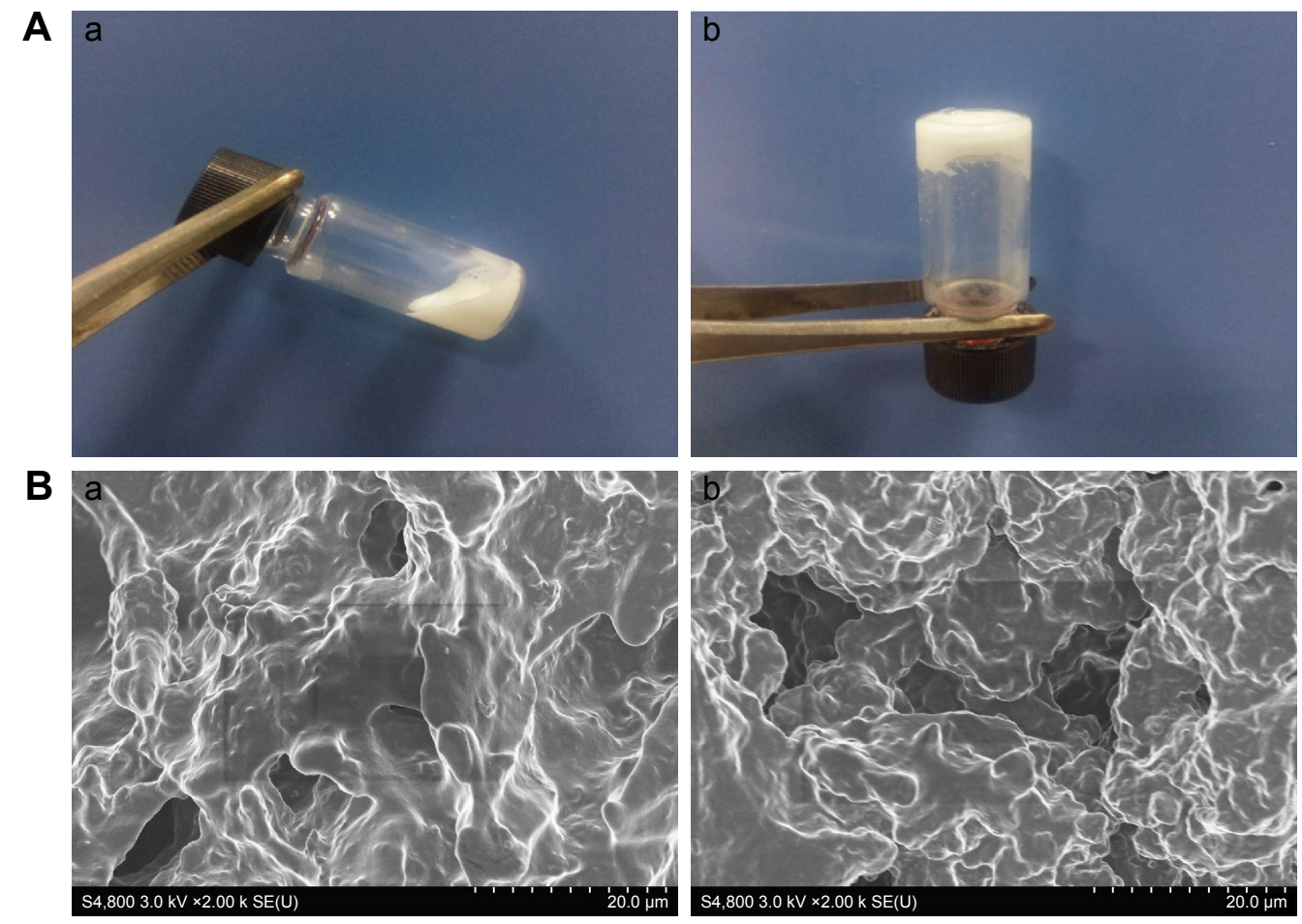

Figure I (A) Images of a) the nanoparticle dispersion sol $\left(25^{\circ} \mathrm{C}\right)$ and b) hydrogel $\left(37^{\circ} \mathrm{C}\right)$ states of bFGF + IBU/PECT NP aqueous dispersions. (B) SEM images of a) lyophilized PECT and b) bFGF + IBU/PECT NP hydrogel $(C p=25 \%)$.

Abbreviations: bFGF, basic fibroblast growth factor; $C_{p}$, nanoparticle mass concentration; IBU, ibuprofen; NP, nanoparticle; PECT, poly( $\varepsilon$-caprolactone-co-I,4,8-trioxa [4.6]spiro-9-undecanone)-poly(ethylene glycol)-poly(ع-caprolactone-co-1,4,8-trioxa [4.6]spiro-9-undecanone); SEM, scanning electron microscopy. 


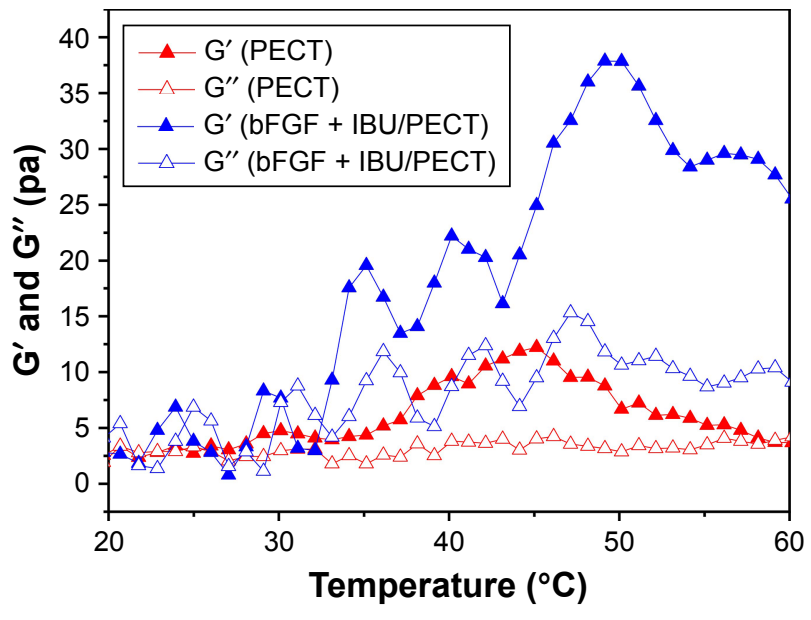

Figure 2 Viscosity of PECT and bFGF + IBU/PECT NP aqueous dispersions as a function of temperature at $25 \%(\mathrm{w} / \mathrm{w})$.

Abbreviations: bFGF, basic fibroblast growth factor; G', storage modulus; G", loss modulus; IBU, ibuprofen; NP, nanoparticle; PECT, poly(E-caprolactone-co-I,4,8trioxa [4.6]spiro-9-undecanone)-poly(ethylene glycol)-poly( $\varepsilon$-caprolactone-co-I,4,8trioxa [4.6]spiro-9-undecanone).

for 48 hours, their cell proliferation levels did not significantly differ from those of the control group $(P>0.05$; Figure 5). Therefore, PECT was not toxic to HGFs and was biocompatible with them.

\section{HGF proliferation}

Immunohistochemical staining of the experimental cells and observation under a light microscope showed that HGF cells were mesodermal in origin and epithelium-free (Figure S1).

We determined that the optimal bFGF concentration for promoting HGF growth was $10 \mathrm{ng} \mathrm{mL}^{-1}$. bFGF had auxiliary HGF-promoting properties at this dosage (Figure S2).

SEM imaging was used to observe the morphology of HGFs adhering to titanium surfaces and to count them. The

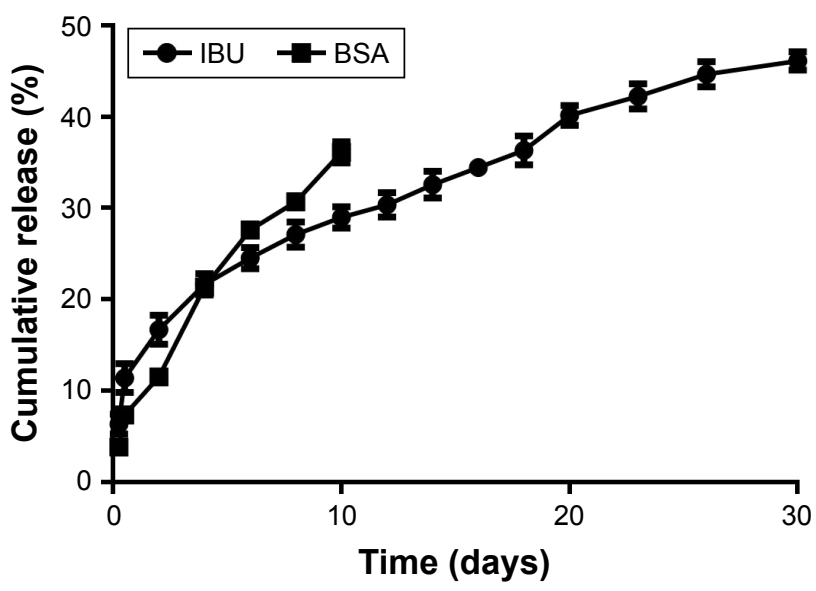

Figure $3 \mathrm{IBU}$ and $\mathrm{BSA}-\mathrm{FITC}$ release profiles at $37^{\circ} \mathrm{C}$ from the hydrogel formulation. Abbreviations: IBU, ibuprofen; FITC, fluorescein isothiocyanate conjugate. images were obtained at magnifications of $\times 500$ and $\times 2,000$. Figure $6 \mathrm{~A}$ shows that there were more HGFs on the titanium disks cultured with bFGF and hydrogel than there were on the control disks. Most of the HGFs in the latter group did not diffuse and were spherical. The HGFs on the titanium discs in bFGF and hydrogel showed protruded pseudopodia and diffused over a large area.

CCK-8 was used to detect HGF proliferation in hydrogel at 1, 3, 5, and 7 days after seeding. As shown in Figure 6B, the absorbances measured for groups $\mathrm{B}$ and $\mathrm{C}$ were significantly different from that of the control $(P<0.05)$. However, groups $\mathrm{B}$ and $\mathrm{C}$ did not differ significantly in terms of HGF proliferation $(P>0.05)$. Therefore, both hydrogel and $b F G F$ promote HGF proliferation equally well.

To examine the long-term effects of hydrogel on HGF proliferation and sustained bFGF release, the Transwell chamber was used to measure absorbances at 1, 3, 5, and 7 days after cell seeding. Figure $6 \mathrm{C}$ shows that the optical densities of groups $\mathrm{B}$ and $\mathrm{C}$ were significantly different from that of the control $(P<0.05)$. The optical density of group $\mathrm{C}$ was higher than that of group $\mathrm{B}$, but the difference was not significant $(P>0.05)$. Therefore, the hydrogel promoted HGF proliferation more than bFGF compared to the control. The effect of hydrogel on HGF proliferation can last $\geq 7$ days (Figure 6C).

\section{HGF adhesion}

Fluorescence microscopy imaging was used to enumerate vinculin proteins and adhered HGFs and to view HGF morphology 48 hours after seeding. As can be seen in Figure 7A, the lowest number of vinculin proteins and adhered HGFs was observed on the control titanium disks. There were more vinculins on the disks in bFGF and hydrogel than there were on the control disks. Moreover, there were more HGFs with superior morphology on the disks in bFGF and hydrogel than there were on the control disks. However, there were no significant differences between the bFGF and hydrogel treatments in terms of numbers of vinculins and HGFs.

qRT-PCR was performed to confirm cell adhesion at the mRNA level. Quantitation of vinculin proteins (Figure 7B) showed that vinculin expression levels were significantly higher in groups $\mathrm{B}$ and $\mathrm{C}$ than in the control $(P<0.01)$. Vinculin expression was significantly higher in the hydrogel group than in the bFGF group $(P<0.05)$.

\section{Anti-inflammatory effect}

ELISA was used to investigate the anti-inflammatory effects of the hydrogel. As shown in Figure 8, the level of PGE2 in group B was significantly higher than that in group A at 

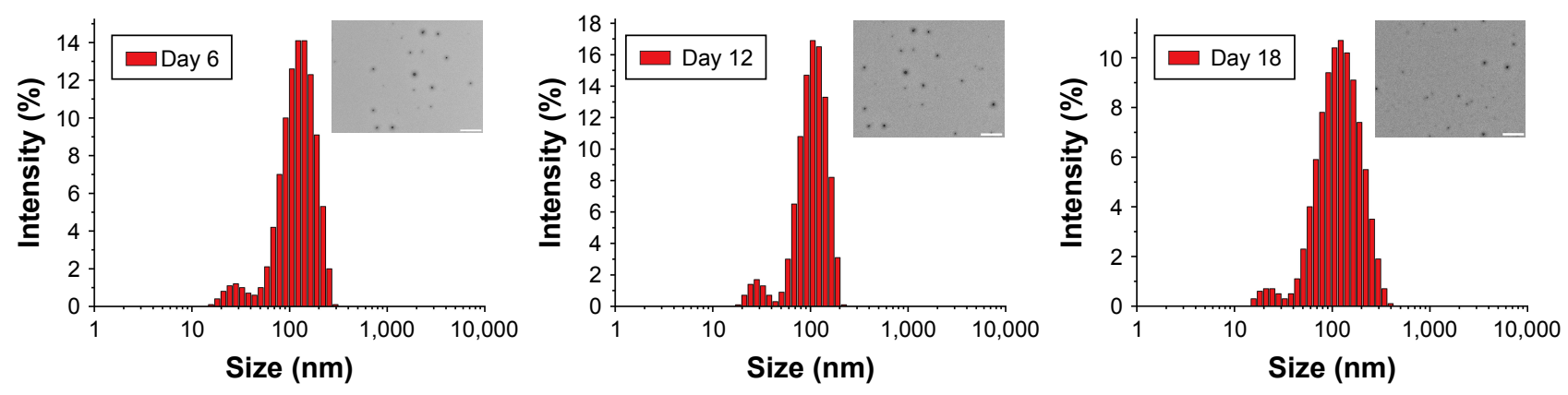

Figure 4 Size distribution of degrading samples during in vitro degradation of bFGF + IBU/PECT NP aqueous dispersions on days 6, I2, and I8. Notes: Illustrations are corresponding TEM images (scale bar: I $\mu \mathrm{m}$ ).

Abbreviations: bFGF, basic fibroblast growth factor; IBU, ibuprofen; NP, nanoparticle; PECT, poly( $\varepsilon$-caprolactone-co-I,4,8-trioxa [4.6]spiro-9-undecanone)-poly(ethylene glycol)-poly(E-caprolactone-co-I,4,8-trioxa [4.6]spiro-9-undecanone); TEM, transmission electron microscopy.

every time point $(P<0.05)$. Thus, $1 \mu \mathrm{g} \mathrm{mL} \mathrm{m}^{-1} \mathrm{Pg}$-LPS induced an inflammatory response in HGFs. Groups $\mathrm{C}$ and D had significantly lower PGE2 levels at 10, 24, 48, and 72 hours than group $\mathrm{B}(P<0.05)$. The level of PGE2 in group D was significantly lower than that in groups $\mathrm{B}$ and $\mathrm{C}$ at every time point $(P<0.05)$. PGE2 decreased with increasing culture time. Therefore, the anti-inflammatory effect of the hydrogel was superior to that of IBU.

\section{Discussion}

Unlike osseointegration, the soft tissues surrounding implants have not been studied much. ${ }^{14-17}$ Soft tissue attachment around the implant neck forms an important barrier to microbes. Marginal gingival tissue can separate the oral microbial environment from the jawbone tissue. The junctional epithelium and the connective tissue adhering to the implant surface constitute this barrier. ${ }^{18}$ The connective

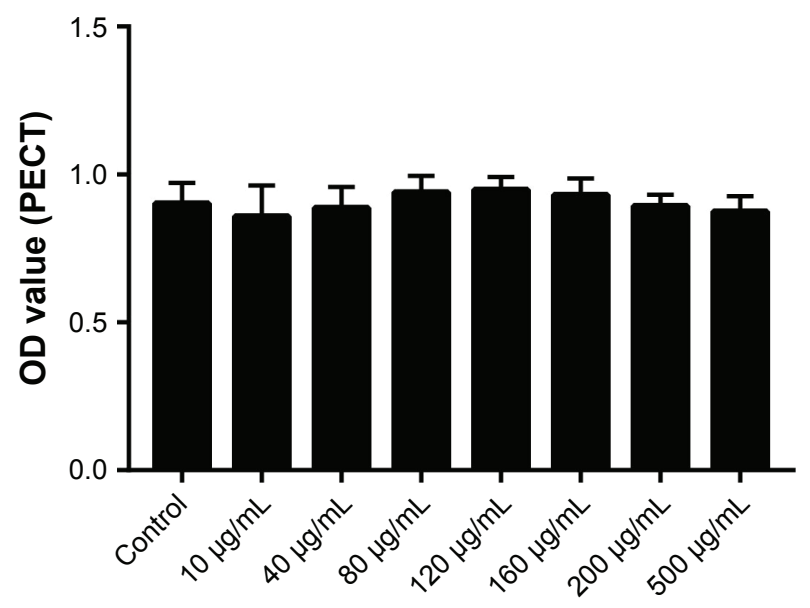

Figure 5 HGF proliferation activity levels at different PECT concentrations after 48 hours.

Abbreviations: HGF, human gingival fibroblast; PECT, poly( $\varepsilon$-caprolactone-coI,4,8-trioxa [4.6]spiro-9-undecanone)-poly(ethylene glycol)-poly( $\varepsilon$-caprolactone-coI,4,8-trioxa [4.6]spiro-9-undecanone). tissue around the implant consists of gingival HGFs and the collagen fibers they secrete, which attach to the implant surface. This process plays an important role in soft tissue healing and directly affects the quality of implant-gingival interface. Dense fibroblasts form a tight adhesion layer around the implant surface and create a biological barrier. If this structure heals well, it can prevent epithelial migration to the fixture, strengthen the edge closure, reduce bacterial invasion, and protect deep bone tissue. Maintenance of healthy soft tissue barriers is as vital as maintaining bone binding itself for the long-term success of implantsupporting prostheses. Reinforcing healing in the implant connective-tissue layer is even more essential for implant soft tissue healing. ${ }^{19}$

Bacterial infection is one of the main causes of implant failure. Local inflammatory response and bacterial infection are the key factors leading to gum tissue destruction. ${ }^{20}$ Although inflammation is an essential part of wound healing, it may hinder tissue regeneration if persistent. Excessive inflammation may impede both wound healing and tissue granulation. ${ }^{21}$ Cyclooxygenase- 2 activation by bacterial stressors and cytokines induces PGE2 biosynthesis in host immune inflammatory mediators. ${ }^{22}$ PGE2 is widely associated with inflammation and bone resorption. It plays an important role in disease progression. ${ }^{23}$ Therefore, developing immunomodulatory strategies may improve periodontal regeneration. Several natural and synthetic compounds have been tested for their reduction of inflammatory markers. ${ }^{24-26}$

As inhibitors of host immune inflammatory mediators, nonsteroidal anti-inflammatory drugs reduce tissue damage and bone loss. ${ }^{27}$ They can also mitigate acute inflammation and pain. ${ }^{28}$ IBU inhibits cyclooxygenase, reduces prostaglandins, and prevents periodontal tissue damage caused by inflammation. ${ }^{29,30}$ It also reduces gingival inflammation and alveolar bone loss. ${ }^{31}$ 


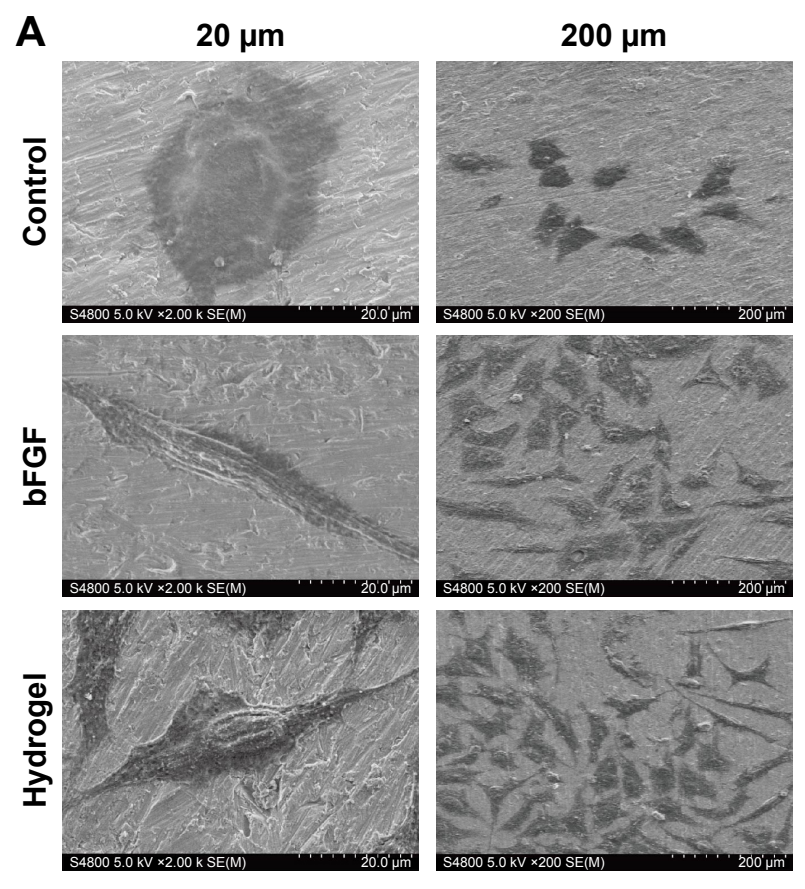

B

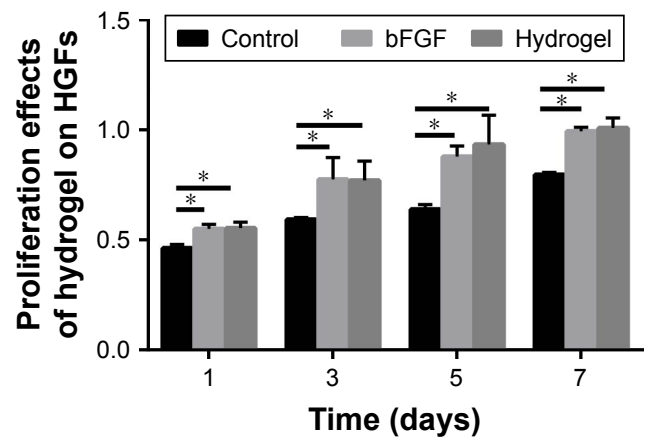

C

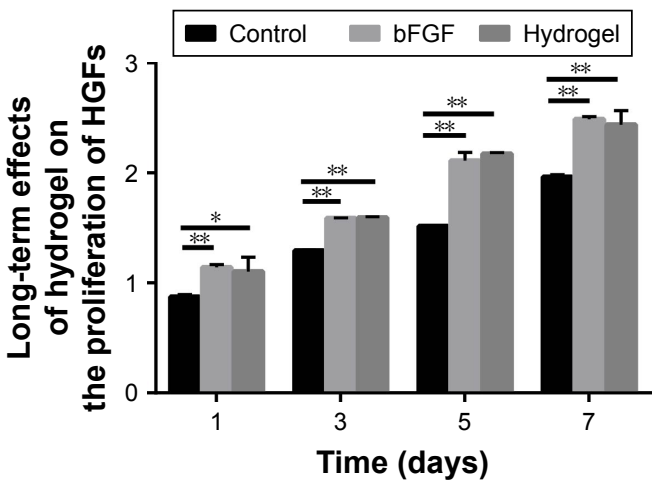

Figure 6 HGF proliferation in vitro.

Notes: (A) SEM of HGFs attached to smooth titanium disk surfaces 48 hours after seeding. (B) HGF proliferation activity in hydrogel at I, 3 , 5, and 7 days. (C) Long-term HGF proliferation activity in hydrogel at I, 3, 5, and 7 days. $* P<0.05 ; * * P<0.01$.

Abbreviations: bFGF, basic fibroblast growth factor; HGF, human gingival fibroblast; SEM, scanning electron microscopy.

In this study, bFGF and IBU were loaded on PECT aiming to promote HGF proliferation and adhesion and suppress inflammation. PECT is a novel temperature-sensitive hydrogel carrier. At room temperature, it is a liquid but rapidly changes into a hydrogel with temperature changes in vivo. Hydrophilicity, viscosity, biocompatibility, and controlled drug release are the key features of successful formulations used for hydrogel drug delivery systems that are injectable in situ. An ideal in situ drug carrier must also achieve high drug-loading efficiency without adversely affecting hydrogelling. ${ }^{32}$ The present study demonstrated the sol-to-hydrogel transition behavior of aqueous polymer dispersions using tube inversion and rheological analysis (Figures 1 and 2). $\mathrm{G}^{\prime \prime}$ increased with temperature more
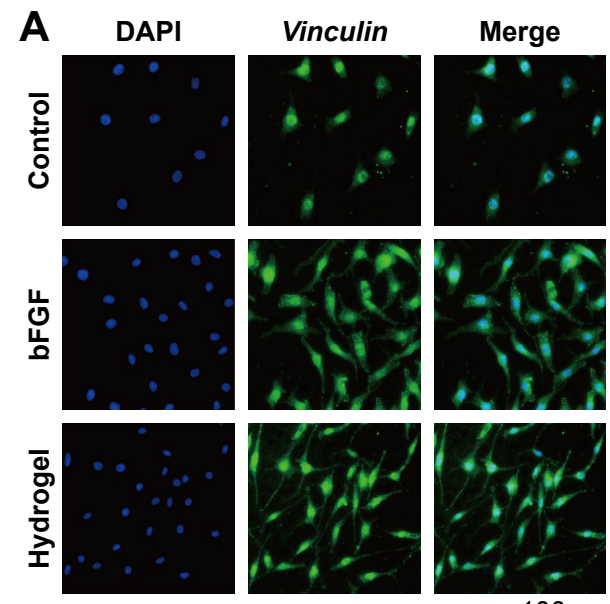

B

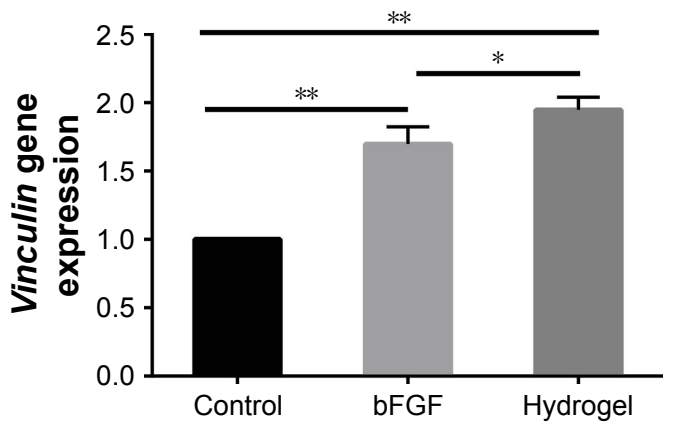

Figure 7 HGF adhesion in vitro.

Notes: (A) Immunofluorescence image of HGFs attached to smooth titanium disk surfaces 48 hours after seeding (green: vinculin proteins; blue: DAPI). (B) qRT-PCR analysis of vinculin genes in HGFs stimulated by hydrogel for 48 hours. $* P<0.05$; $* * P<0.01$.

Abbreviations: bFGF, basic fibroblast growth factor; HGF, human gingival fibroblast; qRT, quantitative real-time. 


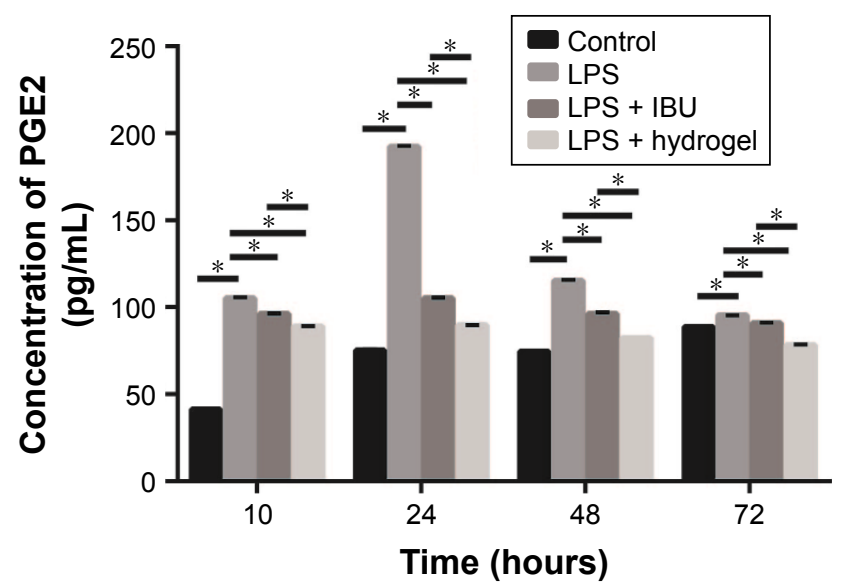

Figure 8 ELISA of PGE2 in HGFs stimulated by hydrogel for 10, 24, 48, and 72 hours. Notes: $* P<0.05$.

Abbreviations: HGF, human gingival fibroblasts; IBU, ibuprofen; LPS, lipopolysaccharide; PGE2, prostaglandin E2.

rapidly than $\mathrm{G}^{\prime}$, indicating that a hydrogel was formed. PECT dispersion proved to be a promising candidate drug delivery system. The hydrogel formulation modulus measured for hydrophilic bFGF and hydrophobic IBU slightly increased in the hydrogel state. Dispersion of PECT loaded with the two drugs changed the modulus in a manner very similar to that of pure PECT. Therefore, both hydrophilic and hydrophobic drugs could be successfully loaded into the hydrogel without affecting its thermosensitivity.

For the controlled delivery of low-molecular weight hydrophilic compounds, high water content and macropores in the hydrogel provide a diffusion pathway conducive to rapid drug release. ${ }^{33}$ Therefore, the two model drugs were separately loaded into the PECT hydrogel and IBU was embedded in its hydrophobic region. In contrast, BSA-FITC is dispersed in the aqueous phase and diffuses easily. Drug release assays in vitro showed that IBU disperses in a nearly linear manner for $\leq 30$ days (Figure 3). BSA-FITC showed a significant release burst in the early stage. As the release kinetics of BSA-FITC and IBU differ substantially, more BSA-FITC than IBU is initially liberated from the double drug-loaded hydrogel. In this way, a high drug concentration is created around the soft tissue and promotes healing there. The release of IBU and the remaining BSA-FITC is prolonged to improve and maintain treatment, and to promote HGF proliferation and adhesion. In addition, they exerted anti-inflammatory effects.

The bFGF + IBU/PECT temperature-sensitive hydrogel integrates the soft tissue healing effect of bFGF and the anti-inflammatory effect of IBU. The bFGF + IBU/PECT hydrogel sustains the release of effective concentrations of bFGF and IBU early in soft tissue healing. In vitro cell experiments showed that the bFGF + IBU/PECT hydrogel enhanced HGF proliferation and vinculin protein expression significantly compared to either bFGF alone or the control. The bFGF + IBU/PECT hydrogel showed a lower Pg-LPS expression level than IBU. Therefore, it had a direct antiinflammatory effect. These data indicate that bFGF + IBU/ PECT hydrogel augments HGF proliferation and adhesion, and protects them from the host immune system. The results of this study indicate that bFGF + IBU/PECT hydrogel may represent a very useful support in postimplant surgery healing and as an early local treatment of peri-implantitis. In the future, we will conduct more in-depth research, such as increasing in vivo experiments and conducting functional studies to exploring specific mechanisms of inflammation regulation to further justify the hydrogel.

\section{Conclusion}

PECT hydrogel loaded with bFGF and IBU promotes HGF proliferation and adhesion and has anti-inflammatory properties. The results confirm that the hydrogel had a suitable controlled-release pattern, sustains the release of both hydrophilic and hydrophobic drugs, promotes HGF proliferation and adhesion, and exerts anti-inflammatory effects. Rational design of the hydrogel tested in the present study may promote soft tissue healing after implant surgery and reduce the risk for peri-implant mucositis.

\section{Acknowledgments}

This study was supported by the 13th 5-Year Comprehensive Investment and First-class Discipline Construction/ Stomatology (No 11601502/xk0126) and Tianjin Medical University Science Fund Project (No 2016KYZM06). The authors thank the Central Laboratory of Tianjin Medical University School of Stomatology.

\section{Disclosure}

The authors report no conflicts of interest in this work.

\section{References}

1. Bernardi S, Gatto R, Severino M, et al. Short versus longer implants in mandibular alveolar ridge augmented using osteogenic distraction: oneyear follow-up of a randomized Split-Mouth trial. J Oral Implantol. 2018; 44(3):184-191.

2. Ma Q, Wang W, Chu PK, et al. Concentration- and time-dependent response of human gingival fibroblasts to fibroblast growth factor 2 immobilized on titanium dental implants. Int J Nanomedicine. 2012;7: 1965-1976.

3. Bernardi S, Bianchi S, Botticelli G, et al. Scanning electron microscopy and microbiological approaches for the evaluation of salivary microorganisms behaviour on anatase titanium surfaces: in vitro study. Morphologie. 2018;102(336):1-6. 
4. Tavares LJ, Pavarina AC, Vergani CE, de Avila ED. The impact of antimicrobial photodynamic therapy on peri-implant disease: what mechanisms are involved in this novel treatment? Photodiagnosis Photodyn Ther. 2017;17:236-244.

5. Inoue $\mathrm{T}$, Takeda $\mathrm{T}$, Lee $\mathrm{CY}$, et al. Immunolocalization of proliferating cell nuclear antigen in the peri-implant epithelium. Bull Tokyo Dent Coll. 1997;38(3):187-193.

6. Kawahara H, Kawahara D, Hashimoto K, Takashima Y, Ong JL. Morphologic studies on the biologic seal of titanium dental implants. Report I. In vitro study on the epithelialization mechanism around the dental implant. Int J Oral Maxillofac Implants. 1998;13(4):457-464.

7. Berglundh T, Lindhe J, Ericsson I, Marinello CP, Liljenberg B, Thomsen P. The soft tissue barrier at implants and teeth. Clin Oral Implants Res. 1991;2(2):81-90.

8. Koka S. The implant-mucosal interface and its role in the long-term success of endosseous oral implants: a review of the literature. Int $J$ Prosthodont. 1998;11(5):421-432.

9. Koutouzis T, Gadalla H, Lundgren T. Bacterial colonization of the implant-abutment interface (IAI) of dental implants with a sloped marginal design: An in-vitro study. Clin Implant Dent Relat Res. 2016;18(1):161-167.

10. Romanos GE, Biltucci MT, Kokaras A, Paster BJ. Bacterial composition at the implant-abutment connection under loading in vivo. Clin Implant Dent Relat Res. 2016;18(1):138-145.

11. Lee J, Stavropoulos A, Susin C, Wikesjö UM. Periodontal regeneration: focus on growth and differentiation factors. Dent Clin North Am. 2010;54(1):93-111.

12. Tian D, Dubois P, Jérôme R. Macromolecular engineering of polylactones and polylactides. 23. Synthesis and characterization of biodegradable and biocompatible homopolymers and block copolymers based on 1,4,8-trioxa[4.6]spiro-9-undecanone. Macromolecules. 1997;30(7):1947-1954.

13. Wang W, Chang L, Li X, et al. Controlled thermal gelation of poly( $(\varepsilon-$ caprolactone)/poly(ethylene glycol) block copolymers by modifying cyclic ether pendant groups on poly( $\varepsilon$-caprolactone). Soft Matter. 2012;8(5):1575-1583.

14. Albrektsson T, Buser D, Sennerby L. On crestal/marginal bone loss around dental implants. Int J Prosthodont. 2012;25(4):320-322.

15. Schwarz F, Iglhaut G, Becker J. Quality assessment of reporting of animal studies on pathogenesis and treatment of peri-implant mucositis and peri-implantitis. A systematic review using the ARRIVE guidelines. J Clin Periodontol. 2012;39(Suppl 12):63-72.

16. Albrektsson T, Buser D, Sennerby L. Crestal bone loss and oral implants. Clin Implant Dent Relat Res. 2012;14(6):783-791.

17. Albrektsson T, Dahlin C, Jemt T, Sennerby L, Turri A, Wennerberg A. Is marginal bone loss around oral implants the result of a provoked foreign body reaction? Clin Implant Dent Relat Res. 2014;16(2): $155-165$.
18. Berglundh T, Lindhe J. Dimension of the periimplant mucosa. Biological width revisited. J Clin Periodontol. 1996;23(10):971-973.

19. Rompen E, Domken O, Degidi M, Pontes AE, Piattelli A. The effect of material characteristics, of surface topography and of implant components and connections on soft tissue integration: a literature review. Clin Oral Implants Res. 2006;17(Supp1 2):55-67.

20. Figuero E, Graziani F, Sanz I, Herrera D, Sanz M. Management of peri-implant mucositis and peri-implantitis. Periodontol 2000. 2014; 66(1):255-273.

21. Hortensius RA, Harley BAC. Naturally derived biomaterials for addressing inflammation in tissue regeneration. Exp Biol Med. 2016; 241(10):1015-1024.

22. Noguchi K, Ishikawa I. The roles of cyclooxygenase- ${ }_{2}$ and prostaglandin E2 in periodontal disease. Periodontol 2000. 2007;43(1):85-101.

23. Krayer JW, Leite RS, Kirkwood KL. Non-surgical chemotherapeutic treatment strategies for the management of periodontal diseases. Dent Clin North Am. 2010;54(1):13-33.

24. Van Dyke TE, Hasturk H, Kantarci A, et al. Proresolving nanomedicines activate bone regeneration in periodontitis. J Dent Res. 2015; 94(1):148-156.

25. Yar M, Farooq A, Shahzadi L, et al. Novel meloxicam releasing electrospun polymer/ceramic reinforced biodegradable membranes for periodontal regeneration applications. Mater Sci Eng C Mater Biol Appl. 2016;64:148-156.

26. Yuan H, Gupte R, Zelkha S, Amar S. Receptor activator of nuclear factor kappa B ligand antagonists inhibit tissue inflammation and bone loss in experimental periodontitis. J Clin Periodontol. 2011;38(11):1029-1036.

27. Howell TH, Williams RC. Nonsteroidal antiinflammatory drugs as inhibitors of periodontal disease progression. Crit Rev Oral Biol Med. 1993;4(2):177-196.

28. Persson GR. Rheumatoid arthritis and periodontitis - inflammatory and infectious connections. Review of the literature. Oral Microbiol. 2012;4(1):11829.

29. Taiyeb Ali TB, Waite IM. The effect of systemic ibuprofen on gingival inflammation in humans. J Clin Periodontol. 1993;20(10):723-728.

30. Jiansheng G. Ibuprofen rescues abnormalities in periodontal tissues in conditional Presenilin 1 and Presenilin 2 double knockout mice. IJMS. 2013;14:18457-18469.

31. Williams RC, Jeffcoat MK, Howell TH, et al. Ibuprofen: an inhibitor of alveolar bone resorption in beagles. J Periodontal Res. 1988;23(4): 225-229.

32. Xu S, Wang W, Li X, Liu J, Dong A, Deng L. Sustained release of PTXincorporated nanoparticles synergized by burst release of $\mathrm{DOX} \cdot \mathrm{HCl}$ from thermosensitive modified PEG/PCL hydrogel to improve antitumor efficiency. Eur J Pharm Sci. 2014;62(4):267-273.

33. Lin CC, Metters AT. Hydrogels in controlled release formulations: network design and mathematical modeling. Adv Drug Deliv Rev. 2006;58(12-13):1379-1408. 


\section{Supplementary materials \\ Materials and methods \\ HGF culture}

HGFs were obtained from two periodontally healthy patients who underwent crown lengthening surgery. All volunteers provided written approval and informed consent. The gingival tissues were triple-washed in PBS containing $100,000 \mathrm{U}$ penicillin/gentamicin. Connective tissues were separated from epithelia using tissue scissors. The tissues were sheared into $1-\mathrm{mm}^{3}$ fragments and uniformly inoculated on the bottom of an 8-mm culture flask at $\sim 6$-mm intervals. The flask was inverted and $3-4 \mathrm{~mL}$ of $10 \% \mathrm{w} / \mathrm{v}$ serum + high-glucose DMEM was added to it. The tissues were incubated for 4-6 hours at $37^{\circ} \mathrm{C}$ under a $5 \% \mathrm{CO}_{2}$ atmosphere. The bottom of the culture flask was slowly rotated until the connective tissue fragments firmly attached to the flask wall. The serum-containing medium was completely infiltrated into the connective tissue block and the incubation continued. After the cells were established, the medium was replaced every 2-3 days. Then $4-5 \mathrm{~mL}$ of $10 \% \mathrm{w} / \mathrm{v}$ FBS + serum + high-glucose DMEM was added to the cell culture. HGFs at passages 4-6 were used in the subsequent analyses.

\section{bFGF cell proliferation assay}

The effects of various bFGF concentrations on HGF proliferation were evaluated with CCK-8 reagent. The bFGF was diluted to five different concentrations using DMEM containing $10 \% \mathrm{w} / \mathrm{v}$ FBS $\left(0.1,1,10,50\right.$, and $\left.100 \mathrm{ng} \mathrm{mL}^{-1}\right)$. HGF cells were seeded into 96-well plates at an initial density of $1 \times 10^{3}$ per well. After 24 hours, the cells were randomly divided into six groups, with five replicates per group. The media for each experimental group were replaced and DMEM containing $10 \% \mathrm{w} / \mathrm{v}$ FBS was used as a blank control. On days 1, 3, 5, and 7, the HGF samples were gently washed with PBS. Media in the wells were replaced with serum-free DMEM $(100 \mu \mathrm{L})$ and CCK-8 solution $(10 \mu \mathrm{L})$. The HGF cells were incubated at $37^{\circ} \mathrm{C}$ for 4 hours. The absorbance of the contents of each well was read by microplate reader at $450 \mathrm{~nm}$.

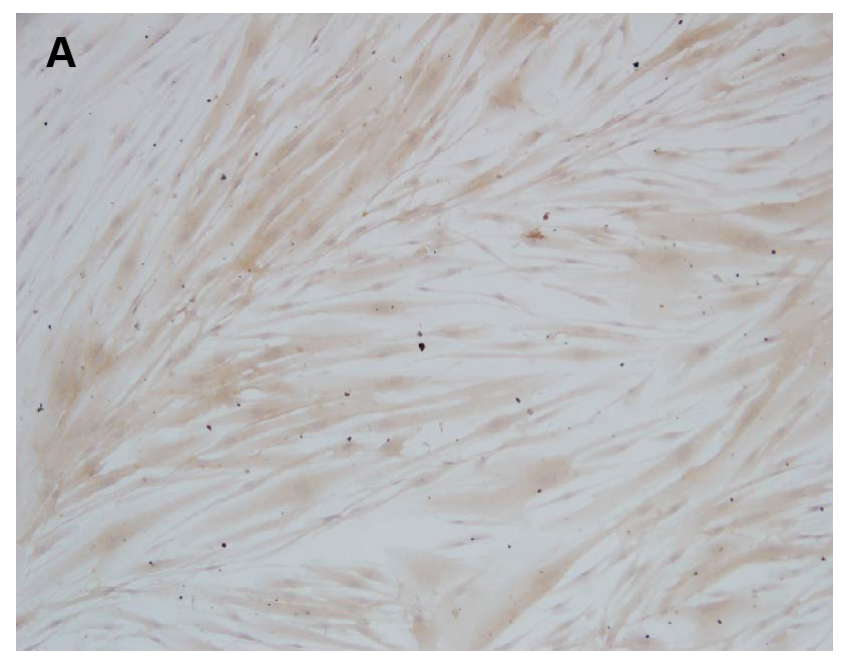

B

Notes: (A) Cells positive for vimentin staining. (B) Cells negative for anti-keratin staining

Abbreviation: HGF, human gingival fibroblast. 


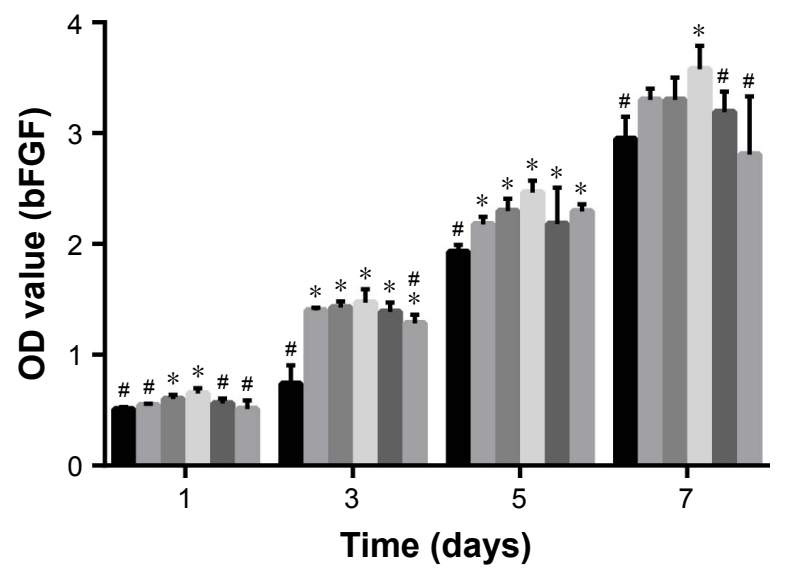

\begin{tabular}{|ll} 
Control & $0.1 \mathrm{ng} / \mathrm{mL}$ bFGF \\
$1 \mathrm{ng} / \mathrm{mL}$ bFGF & $50 \mathrm{ng} / \mathrm{mL} \mathrm{bFGF}$ \\
$10 \mathrm{ng} / \mathrm{mL}$ bFGF & $100 \mathrm{ng} / \mathrm{mL} \mathrm{bFGF}$
\end{tabular}

Figure S2 HGF proliferation activity at various bFGF concentrations at I, 3, 5, and 7 days.

Notes: *Represents $P<0.05$ compared with the control group, " represents $P<0.05$ compared with the $10 \mathrm{ng} \mathrm{mL} \mathrm{mroup}^{-1}$.

Abbreviations: bFGF, basic fibroblast growth factor; HGF, human gingival fibroblast.

\section{Publish your work in this journal}

The International Journal of Nanomedicine is an international, peerreviewed journal focusing on the application of nanotechnology in diagnostics, therapeutics, and drug delivery systems throughout the biomedical field. This journal is indexed on PubMed Central, MedLine, CAS, SciSearch $®$, Current Contents ${ }^{\circledR} /$ Clinical Medicine,
Journal Citation Reports/Science Edition, EMBase, Scopus and the Elsevier Bibliographic databases. The manuscript management system is completely online and includes a very quick and fair peer-review system, which is all easy to use. Visit http://www.dovepress.com/ testimonials.php to read real quotes from published authors. 